\title{
Rare imaging appearance of adenoid cystic carcinoma of the breast: A case report
}

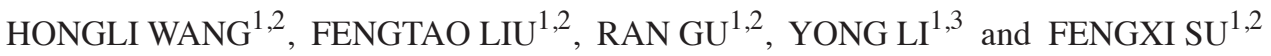 \\ ${ }^{1}$ Guangdong Provincial Key Laboratory of Malignant Tumor Epigenetics and Gene Regulation, Sun Yat-sen Memorial \\ Hospital, Sun Yat-sen University, Guangzhou, Guangdong 510120; ${ }^{2}$ Breast Tumor Center and ${ }^{3}$ Department \\ of Radiology, Sun Yat-sen Memorial Hospital, Sun Yat-sen University, Guangzhou, Guangdong 510288, P.R. China
}

Received February 3, 2017; Accepted May 26, 2017

DOI: $10.3892 /$ mco.2017.1322

\begin{abstract}
Adenoid cystic carcinoma (ACC) of the breast is a rare tumor comprising $<0.1 \%$ of all breast malignancies. The present study reports the case of a 66-year-old woman who underwent multiple imaging techniques, including mammography, ultrasonography and magnetic resonance imaging (MRI). The patient reported gradual enlargement of a mass in the left breast. Mammography identified a high-density, irregular mass, with nipple retraction. Ultrasonography revealed an irregular, complex cystic and solid mass with an unclear margin in the subareolar region. The internal vascularity was seen on Doppler examination. The lesion on MRI was also mixed cystic and solid, with an irregular shape and irregular margins. The solid part of the lesion appeared heterogeneously isointense on T1-weighted imaging (WI) and a slightly high signal on T2WI, and exhibited internal septations with a low signal on T2WI. Following injection of contrast agent, the enhancement of the solid part was rapid and heterogeneous. The cystic parts of lesion appeared as high-signal on T1WI and T2WI. The tumor infiltrated the left nipple and adjacent skin. The patient underwent a left modified radical mastectomy with axillary lymph node dissection. The pathological examination confirmed the tumor as ACC of the left breast. The axillary lymph nodes were negative for tumor metastasis. Therefore, the differential diagnosis of mixed cystic and solid breast masses with unclear margins and internal septations on T2WI exhibiting delayed enhancement, must include ACC.
\end{abstract}

Correspondence to: Dr Fengxi Su, Breast Tumor Center, Sun Yat-sen Memorial Hospital, Sun Yat-Sen University, 33 Yingfeng Road, Haizhu, Guangzhou, Guangdong 510288, P.R. China

E-mail: fengxisu@vip.163.com

Abbreviations: ACC, adenoid cystic carcinoma; MRI, magnetic resonance imaging; ER, estrogen receptor; HER2, human epidermal growth factor receptor 2; PR, progesterone receptor; T1WI, T1-weighted imaging; T2WI, T2-weighted imaging

Key words: adenoid cystic carcinoma, breast, mammography, ultrasonography, magnetic resonance imaging

\section{Introduction}

Adenoid cystic carcinoma (ACC) usually occurs in salivary glands, but it has also been described in other organs, including the breast, trachea, uterine cervix, larynx and Bartholin's glands (1-5). However, ACC of the breast is rare (6); thus, there are only a few published studies describing the findings of multiple imaging techniques, and non-specific imaging characteristics have been found on mammography and ultrasonography $(7,8)$. The aim of this study was to present a rare case of proven ACC of the breast and describe its imaging characteristics.

\section{Case report}

In April 2016, a 66-year-old woman was admitted to the Sun Yat-sen Memorial Hospital (Guangzhou, China) with a mass in the left breast that had been present for 4 years. The patient reported gradual enlargement of this mass over the last 2 months. Breast examination revealed a palpable mass in the subareolar region measuring $5.0 \times 5.0 \mathrm{~cm}$, with nipple retraction. There was no abnormal nipple discharge or skin redness. Axillary and subclavicular lymph nodes were not palpable. Mammography identified a $6.8 \times 5.0 \mathrm{~cm}$, high-density, irregular mass, with nipple retraction (Fig. 1). Ultrasonography revealed an irregular mass sized $5.0 \times 4.3 \times 5.0 \mathrm{~cm}$, without a clearly circumscribed margin, in the subareolar region. The internal echo of the mass was mixed cystic and solid, with partial enhancement of the posterior echo (Fig. 2). On Doppler examination, internal vascularity was seen. The patient underwent a contrast-enhanced bilateral breast magnetic resonance imaging (MRI) examination in the prone position. The MRI revealed a left breast mass sized $6.3 \times 4.6 \times 6.2 \mathrm{~cm}$. The mass appeared to have an irregular shape and irregular margins. The lesion was mixed cystic and solid. Compared with normal glandular tissue, the solid part of the lesion appeared heterogeneously isointense on T1-weighted imaging (WI; Fig. 3A), with a slightly high signal on T2WI (Fig. 3B). Furthermore, the solid part of the lesion included internal septations with a low signal on T2WI (Fig. 3B). Following injection of contrast agent, the enhancement of the solid component was rapid and heterogeneous (Fig. 3C). The cystic parts of lesion appeared as high-signal on T1WI and T2WI, and two layers were observed 


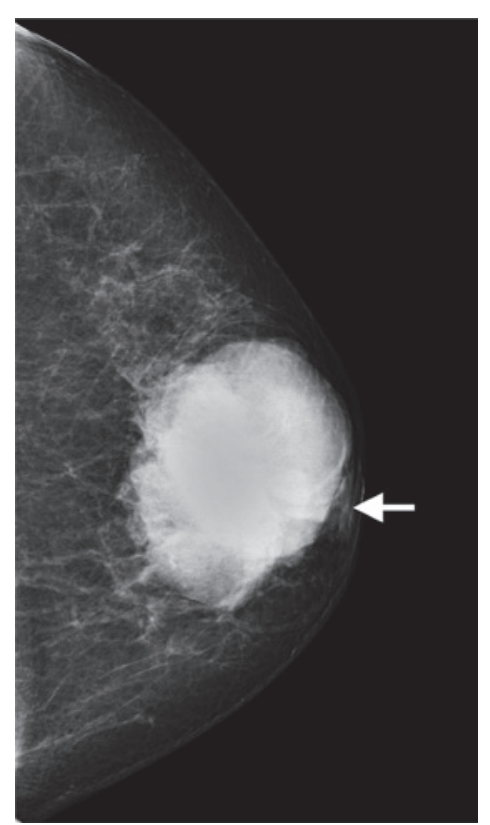

Figure 1. A mammography image showing a high-density, irregular mass in the subareolar region, with nipple retraction (arrow).

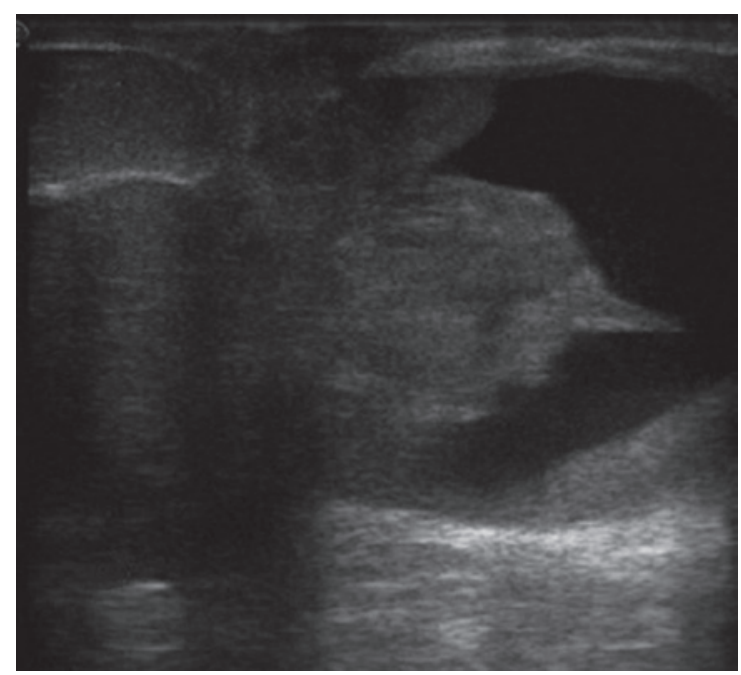

Figure 2. Ultrasonographic image showing a mixed cystic and solid mass without a well-circumscribed margin.

in the largest cystic part. The tumor infiltrated the left nipple and adjacent skin.

Ultrasound-guided 14-gauge core biopsy was performed with a BARD biopsy instrument. On microscopic examination, the tumor cells were arranged in a tubular or cribriform pattern, and exhibited consistent size, small nuclei and nuclear fission. The findings of the histopathological evaluation of the specimens conformed to those of invasive carcinoma, and suggested the possibility of ACC. On immunohistochemistry, the tumor cells were negative for progesterone receptor (PR) and human epidermal growth factor receptor 2 (HER2), but positive for estrogen receptor (ER) (focally, $1 \%$ positive) and CD117. Due to the large size of the mass, the patient underwent left modified radical mastectomy with axillary lymph node dissection. The breast specimen measured $17 \times 14 \times 5.0 \mathrm{~cm}$ and contained a mixed cystic and solid, poorly defined mass measuring
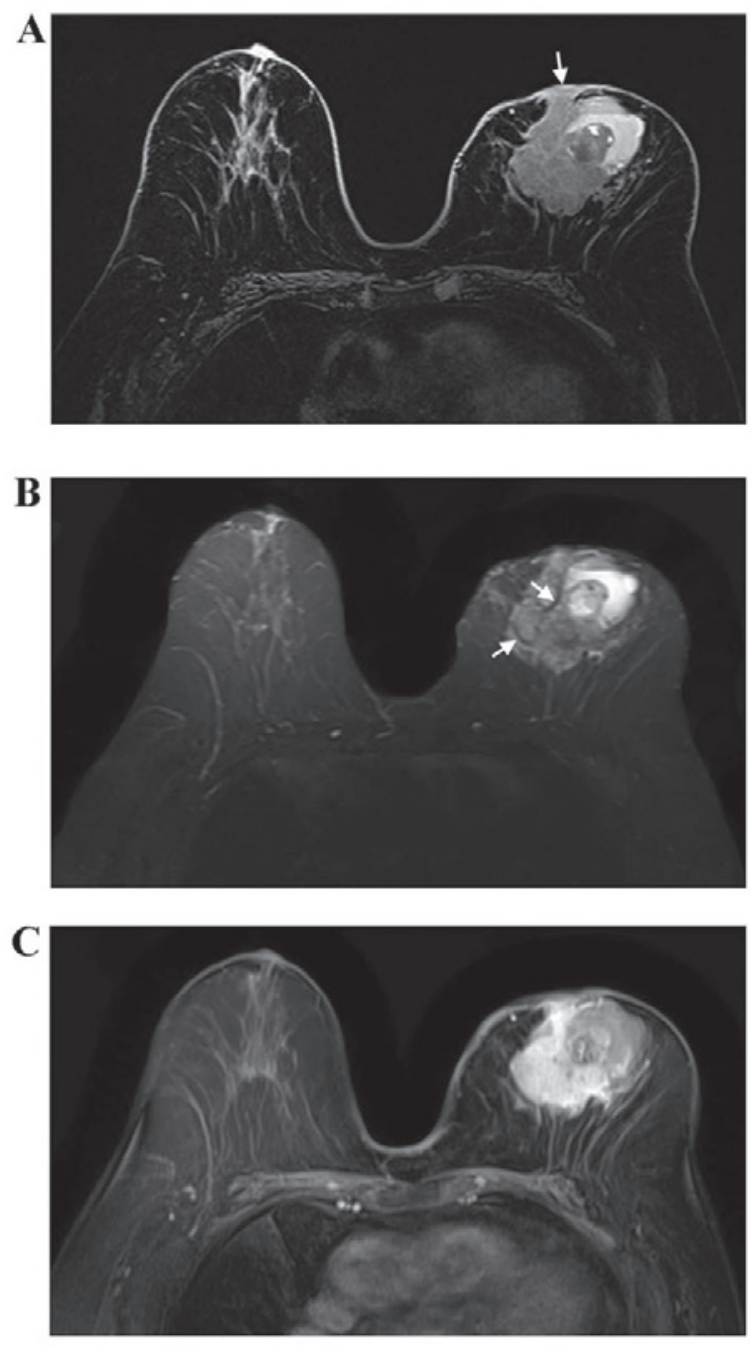

Figure 3. (A) The solid part of lesion appeared heterogeneous isointense on T1WI. The cystic parts of lesion were high signal on T1WI, two layers were seen in the largest cystic part. Tumor infiltrated left nipple and adjacent skin (arrow). (B) The cystic parts of lesion were high signal on T2WI. The solid part of lesion appeared slightly high signal and had internal septations with low signal (arrow) on T2WI. (C) The enhancement of the solid part was rapid and heterogeneous.

$4.5 \times 4.5 \times 3.0 \mathrm{~cm}$. The solid part of the lesion was grey-white and grey-red. Grey-red papillary projections and a hematoma were observed in the cystic cavity. The pathological examination of the specimen identified the tumor as ACC of the left breast. The resected lymph nodes were negative for tumor metastasis. Since the ER was $1 \%$ positive, treatment with tamoxifen $(20 \mathrm{mg} /$ day $)$ for 5 years was decided upon, but was discontinued after 2 months, as the patient was unable to afford treatment. No chemotherapy or radiotherapy have been performed to date. At the last follow-up visit (May 29, 2017), the patient remained free of locoregional recurrence and distant metastases. The patient provided a signed informed consent regarding the publication of the case details and associated images.

\section{Discussion}

ACC was first described by Billroth in 1856 and was characterized as 'cylindroma' (9). Geschickter and Copeland referred to this tumor as 'adenocystic basal cell cancer of the 
breast' in 1945, acknowledging its eccrine gland origin and slow growth (10). ACC of the breast is a particularly rare tumor, comprising $<0.1 \%$ of all breast malignancies (6), and it occurs mainly in women during their fifth and sixth decades of life (11). Consistent with the current literature, our patient was 66-year-old. The most common clinical manifestation of ACC of the breast is a soft mass, commonly located in the subareolar area (6), as was the case in our patient. Multiple cytoarchitectural patterns have been reported (cribriform, tubular, trabecular and solid), and a mixture of different growth patterns is generally observed (12). In the present case, the tumor cells exhibited tubular or cribriform arrangement. ACC of the breast is frequently negative for ER and PR, as well as HER2 gene amplification (triple-negative) (6). However, ER-positive and PR-positive ACC has also been reported (13). In the present study, the patient was PR- and HER2-negative, but focally (1\%) positive for ER. The significance of the positive hormone receptor status is not known, and it may be associated with a non-pure ACC or an invasive cribriform carcinoma with a significantly worse prognosis. The tumor in the present case exhibited diffuse CD117 positivity. CD117 is typically positive in ACC, and it is used to differentiate ACC from conventional breast carcinomas (14). ACC has been reported to have an excellent survival rate, despite its malignant nature, with distant metastases and involvement of the axillary nodes being exceedingly rare (15).

On mammography, the majority of the ACCs of the breast present as high-density masses with irregular or lobulated shape and indistinct margins, which have been described as malignant-appearing $(8,16-19)$; these characteristics were consistent with our findings. The sonographic appearance of ACC has been described as a hypoechoic or heterogeneous mass with irregular, lobulated or oval contours and unclear margin $(7,8,18)$. In the present case, the shape and margins of the mass were consistent with previously reported cases, while the echo of the mass was different. The internal echo of the mass in the present case was mixed cystic and solid. One previous case also reported small cysts within in a rounded nodule (18).

The MRI appearance of ACC of the breast remains controversial. Glazebrook et al (8) found that, on MRI, the masses appeared to have a lobulated or irregular shape, with spiculated margins. Two larger masses exhibited extensive hyperintensity on T2WI, with rapid and heterogeneous enhancement, which was consistent with our case. However, Tsuboi et al (20) and Tang et al (7) found that the majority of the cases had a benign appearance. In addition, Tang et al (7) found that all masses had internal septations with low signal on T2WI, and that the internal septations in the larger masses were enhanced in the delayed phase. Internal septations on T2WI were also observed in the present case, but exhibited heterogeneous enhancement. Tang et al (7) hypothesized that the internal septations observed on T2WI may correspond to stroma being embedded within the tumor islands. The internal septations may help to distinguish ACC of the breast from infiltrating ductal carcinoma and fibroadenoma of the breast. Some fibroadenomas display non-enhancing internal septations, while the internal septations of ACC have been described as delayed-enhanced, particularly in the larger lesions. Furthermore, the mass in the present study was mixed cystic and solid, and the cystic parts of lesion contained hematomas, which has not been previously reported.
In conclusion, $\mathrm{ACC}$ of the breast is a rare type of breast cancer, which may be difficult to distinguish from other types of breast lesions on mammography. The following signs may suggest the diagnosis: mixed cystic and solid mass with unclear margin on ultrasonography and MRI, and internal septations on T2WI that exhibit delayed enhancement on MRI (7). A core biopsy may be required to confirm the diagnosis.

\section{References}

1. Cavanzo FJ and Taylor HB: Adenoid cystic carcinoma of the breast. An analysis of 21 cases. Cancer 24: 740-745, 1969.

2. Cleveland RH, Nice CM Jr and Ziskind J: Primary adenoid cystic carcinoma (cylindroma) of the trachea. Radiology 122: 597-600, 1977.

3. Prempree T, Villasanta U and Tang CK: Management of adenoid cystic carcinoma of the uterine cervix (cylindroma): Report of six cases and reappraisal of all cases reported in the medical literature. Cancer 46: 1631-1635, 1980.

4. Olofsson $\mathrm{J}$ and van Nostrand AW: Adenoid cystic carcinoma of the larynx: A report of four cases and a review of the literature. Cancer 40: 1307-1313, 1977.

5. Addison A and Parker RT: Adenoid cystic carcinoma of Bartholin's gland: A review of the literature and report of a patient. Gynecol Oncol 5: 196-201, 1977.

6. Boujelbene N, Khabir A, Boujelbene N, Jeanneret Sozzi W, Mirimanoff RO and Khanfir K: Clinical review-breast adenoid cystic carcinoma. Breast 21: 124-127, 2012.

7. Tang W, Peng WJ, Gu YJ, Zhu H, Jiang TT and Li C: Imaging manifestation of adenoid cystic carcinoma of the breast. J Comput Assist Tomogr 39: 523-530, 2015.

8. Glazebrook KN, Reynolds C, Smith RL, Gimenez EI and Boughey JC: Adenoid cystic carcinoma of the breast. AJR Am J Roentgenol 194: 1391-1396, 2010.

9. Billroth T: Studies on the Development of the Blood Vessels Combined with Observations from the Royal Surgical University Clinic of Berlin. Georg Reimer, Berlin, pp55-69, 1856 (In German).

10. Geschickter CF and Copeland MM: Diseases of the Breast: Diagnosis, Pathology, Treatment. 2nd edition. J B Lippincott, Philadelphia, pp421-422, 1945.

11. Ghabach B, Anderson WF, Curtis RE, Huycke MM, Lavigne JA and Dores GM: Adenoid cystic carcinoma of the breast in the United States (1977 to 2006): A population-based cohort study. Breast Cancer Res 12: R54, 2010.

12. Da Silva L, Buck L, Simpson PT, Reid L, McCallum N, Madigan BJ and Lakhani SR: Molecular and morphological analysis of adenoid cystic carcinoma of the breast with synchronous tubular adenosis. Virchows Arch 454: 107-114, 2009.

13. Arpino G, Clark GM, Mohsin S, Bardou VJ and Elledge RM: Adenoid cystic carcinoma of the breast: Molecular markers, treatment, and clinical outcome. Cancer 94: 2119-2127, 2002.

14. Mastropasqua MG, Maiorano E, Pruneri G, Orvieto E, Mazzarol G, Vento AR and Viale G: Immunoreactivity for c-kit and p63 as an adjunct in the diagnosis of adenoid cystic carcinoma of the breast. Mod Pathol 18: 1277-1282, 2005.

15. Peters GN and Wolff M: Adenoid cystic carcinoma of the breast. Report of 11 new cases: Review of the literature and discussion of biological behavior. Cancer 52: 680-686, 1983.

16. Youk JH, Kim MJ, Kim EK, Lee JY, Oh KK and Park BW: Recurrence of adenoid cystic carcinoma in the breast after lumpectomy and adjuvant therapy. J Ultrasound Med 25: 921-924, 2006.

17. Ichikawa K, Mizukami Y, Takayama T, Takemura A, Miyati T and Taniya T: A case of adenoid cystic carcinoma of the breast. J Med Ultrason (2001) 34: 193-196, 2007.

18. de Luis E, Apesteguía L, Noguera JJ,Pina L, Martínez-Regueira F, Miguel C and Sáenz J: Adenoid cystic carcinoma of the breast. Radiologia 48: 235-240, 2006 (In Spanish).

19. Santamaría G, Velasco M, Zanón G, Farrús B, Molina R, Solé M and Fernández PL: Adenoid cystic carcinoma of the breast: Mammographic appearance and pathologic correlation. AJR Am J Roentgenol 171: 1679-1683, 1998.

20. Tsuboi N, Ogawa Y, Inomata T, Nishioka A, Yoshida D, Yoshida S and Moriki T: Dynamic MR appearance of adenoid cystic carcinoma of the breast in a 67-year-old female. Radiat Med 16: 225-228, 1998. 